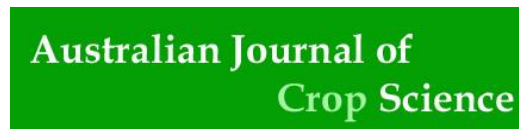

\title{
Genetic diversity among cultivated and wild lentils for iron, zinc, copper, calcium and magnesium concentrations
}

\author{
Debjyoti Sen Gupta ${ }^{1,2, *}$, Dil Thavarajah ${ }^{3, *}$, Rebecca J. McGee ${ }^{4}$, Clarice J. Coyne ${ }^{5}$, Shiv Kumar ${ }^{6}$, \\ Pushparajah Thavarajah ${ }^{7}$
}

${ }^{1}$ Cereal Science Graduate Program, Department of Plant Sciences, North Dakota State University, Fargo, ND, 58102, USA

${ }^{2}$ ICAR-ARS, Division of Crop Improvement, Indian Institute of Pulses Research, Kanpur, UP, 208024, India, ${ }^{3}$ Department of Plant \& Environmental Sciences, 130 McGinty Court, 270 Poole Agricultural Center, Clemson University, Clemson, SC 29634, USA

${ }^{4}$ Grain Legume Genetics and Physiology Research Unit, USDA/ARS, Washington State University, Pullman, WA 99164, USA

${ }^{5}$ Western Regional Plant Introduction Station, USDA/ARS, Washington State University, Pullman, WA 99164, USA

${ }^{6}$ BIGM Program, ICARDA, B. P. 6299, Rabat-Institutes, Rabat, Morocco

${ }^{7}$ International College Beijing, China Agricultural University, No.17 Qinghua Dong Road, Haidian District, Beijing, Post Code 100083, China

*Corresponding authors: debgpb@gmail.com, dthavar@clemson.edu

\begin{abstract}
Information on the seed mineral concentration of cultivated and wild lentils is limited. The objective of this study was to determine the concentrations of iron $(\mathrm{Fe})$, zinc $(\mathrm{Zn})$, calcium $(\mathrm{Ca})$, copper $(\mathrm{Cu})$, and magnesium $(\mathrm{Mg})$ in the seeds of 26 lentil genotypes representing 4 species and 3 subspecies of Lens. Plants were grown in a greenhouse using a completely randomized design with three replicates $(\mathrm{n}=78)$. Seed mineral concentrations were measured using acid digestion followed by inductively coupled plasma-optical emission spectroscopy. Concentrations of $\mathrm{Fe}, \mathrm{Zn}, \mathrm{Ca}, \mathrm{Cu}$, and $\mathrm{Mg}$ in seeds varied from 26-92, 17-51, 97-536, 3-12 and 272-892 mg $\mathrm{kg}^{-1}$, respectively, among the Lens culinaris genotypes. Mineral concentrations for L. lamottei $(\mathrm{Fe}=64-80, \mathrm{Zn}=26-40, \mathrm{Ca}=311-434$, $\left.\mathrm{Cu}=2-6, \mathrm{Mg}=754-839 \mathrm{mg} \mathrm{kg}^{-1}\right)$, L. nigricans $\left(60-70,33-39,508-590,3-4,445-738 \mathrm{mg} \mathrm{kg}^{-1}\right)$ and L. ervoides $(65,37,339,6,638 \mathrm{mg}$ $\mathrm{kg}^{-1}$ ) were within the range of Lens culinaris genotypes. No wild species was superior to cultivated ones for all micronutrients. A larger set of germplasm should be evaluated in future experiments to identify additional genetic variation in lentil for these micronutrients.
\end{abstract}

Keywords: Lens species, minerals, micronutrients, nutrition, germplasm characterization, phenotyping.

Introduction

Two billion people aroundthe world suffer from micronutrient malnutrition (IFAD/FAO/WFP, 2011). Micronutrient deficiency results from an inadequate intake of vitamins and minerals in people's diets. Different methods are available today to prevent micronutrient malnutrition, including food fortification, dietary supplementation, diet diversification, and biofortification. Biofortification, using traditional plant breeding practices combined with biotechnology, is a sustainable approach to the development of mineral-dense staple crops (Pfeiffer and McClafferty, 2007; Welch and Graham, 2004). Biofortification has been a success for several staple food crops and high protein maize (Zea mays L.) (QPM), $\beta$-carotene-rich sweet potato (Ipomoea batatas) and rice (Oryza sativa), and iron-rich commonbean (Phaseolus vulgaris) and pearlmillet (Pennisetum glaucum) cultivars aregrown in countries in Asia and Africa (Bouis et al., 2013). White and Broadley, (2009) reviewed different mineral biofortification research activities in various crops. They highlighted the potential of agronomic as well as the genetic biofortication to improve the availability of seven mineral nutrients in the human diet, namely, iron $(\mathrm{Fe})$, zinc $(\mathrm{Zn})$, copper $(\mathrm{Cu})$, calcium $(\mathrm{Ca})$, magnesium $(\mathrm{Mg})$, iodine $(\mathrm{I})$ and selenium (Se) (White and Broadley, 2009).The development of biofortified crop varieties, particularly nutrient rich food legumes, would have a positive impact in alleviating mineral malnutrition in Asian and African nations. Lentil (Lens culinaris Medik.) is a popular pulse crop, grown and consumed throughout the world. Lens is a small genus belonging to the Fabaceae family of the Viciae tribe. The genus contains one cultivated species ( $L$. culinaris subsp. culinaris) with four subspecies (L. culinaris subsp. culinaris, L.culinaris subsp. orientalis, L. culinaris subsp. odemensis and $L$. culinaris subsp. tomentosus) and three wild species ( $L$. ervoides, L. nigricans, and L. lamottei) (Ferguson et al., 2000). Lentil is a potential candidate for mineral biofortification as its nutritional profile is rich in $\mathrm{Fe}, \mathrm{Zn}$, and Se (Thavarajah et al., 2011; USDA National Nutrient Database, 2015). Identification of mineral dense lentil 
genotypes is a priority for biofortification research. Karaköy et al., (2012) evaluated mineral concentration of a set of Turkish landraces and cultivated genotypes of lentil and reported considerable variability for $\mathrm{Fe}, \mathrm{Zn}, \mathrm{Cu}$, calcium (Ca), and $\mathrm{Mg}$ concentrations. Alghamdi et al., (2014) evaluated 35 advanced ICARDA breeding lines in Saudi Arabia at one field location over two seasons and reported significant variation for $\mathrm{Fe}, \mathrm{Zn}, \mathrm{Cu}, \mathrm{Ca}, \mathrm{Mg}$, phosphorus $(\mathrm{P})$, potassium $(\mathrm{K})$, and manganese $(\mathrm{Mn})$ concentrations. However, there is limited information regarding the variation in mineral concentrations among the subspecies of $L$. culinaris and the wild relatives. If high mineral concentrations exist in the subspecies or wild relatives, interspecific hybridization could be used to introgress alleles associated with improved nutritional quality into cultivated lentil (Ladizinsky, 1985). The Lens subspecies and wild relatives are routinely utilized in breeding programs as sources of novel traits, such as disease resistance, not found in cultivated lentil (Fialaet al., 2009). In order to identify potential candidate donors, lentil and its wild relatives need to be evaluated to determine the variability for mineral concentrations. The objective of this study was to determine the seed mineral concentrations of 26 Lens genotypes grown under greenhouse conditions.

\section{Results}

\section{Iron concentration in cultivated and wild lentils}

Mean Fe concentration was $61 \mathrm{mg} \mathrm{kg}^{-1}$ across all 26 lentil genotypes tested (Table 2). Among the $20 \mathrm{~L}$. culinaris genotypes, Fe concentration ranged from 26 (IG72830) to 92 $\mathrm{mg} \mathrm{kg}^{-1}$ (CDC Red Rider) with a mean of $58 \mathrm{mg} \mathrm{kg}^{-1}$. CDC Redberry and CDC Red Rider had significantly higher concentration of $\mathrm{Fe}$ compared to other tested genotypes. Fe concentration was significantly lower in the genotypes belonging to different L.culinaris subspecies (L.culinaris subsp. culinaris, L.culinaris subsp. orientalis, and $L$. culinaris subsp. tomentosus) than in improved cultivars or breeding lines (L.culinarissubsp.culinaris). L.lamottei genotype IG110810 had a significantly higher concentration $\left(80 \mathrm{mg} \mathrm{kg}^{-1}\right)$ of $\mathrm{Fe}$ compared to other non-culinaris wild types. All the non-culinaris wild type genotypes differed significantly $(P<0.05)$ in terms of Fe concentration except IG110812 (L. lamottei) and IG72815 (L. lervoides). Percent recommended dietary allowance (RDA) of $\mathrm{Fe}$ for the genotypes evaluated ranged from $14-51 \%$ per serving.

\section{Zinc concentration in cultivated and wild lentils}

For the 26 lentil genotypes evaluated, the mean $\mathrm{Zn}$ concentration was $33 \mathrm{mg} \mathrm{kg}^{-1}$ (Table 2). $\mathrm{Zn}$ concentration ranged from 17 (IG72830) to $51 \mathrm{mg} \mathrm{kg}^{-1}$ (CDC Rosetown) among the $20 \mathrm{~L}$. culinaris genotypes with a mean of $32 \mathrm{mg}$ $\mathrm{kg}^{-1}$ (Table 2). Within L. culinaris subsp. culinaris genotypes CDC Rosetown (51 mg kg-1) had significantly higher concentration of $\mathrm{Zn}$ compared to other genotypes. Among the other subspecies (L. culinaris subsp. culinaris, L. culinaris subsp. orientalis, and L. culinaris subsp. tomentosus) IG72614 had significantly higher Zn concentration (43 mg $\mathrm{kg}^{-1}$ ) compared to other genotypes. All the non-culinaris wild type genotypes differed significantly in terms of $\mathrm{Zn}$ concentration. Among the non-culinaris wild types IG110812 had significantly higher concentration of $\mathrm{Zn}\left(40 \mathrm{mg} \mathrm{kg}^{-1}\right)$. Each serving of lentil accounts for 21-64\% of RDA of $\mathrm{Zn}(8$ mg) (Otten et al., 2006).

\section{Copper concentration in cultivated and wild lentils}

The mean $\mathrm{Cu}$ concentration across all lentil genotypes was 6 mg $\mathrm{kg}^{-1}$ (Table 3). $\mathrm{Cu}$ concentration among the $20 \mathrm{~L}$. culinaris genotypes ranged from 2.6 (IG72688) to $12.0 \mathrm{mg}$ $\mathrm{kg}^{-1}$ (CDC Rosetown) with a mean of $6 \mathrm{mg} \mathrm{kg}^{-1}$. Within $L$. culinaris subsp. culinaris genotypes CDC Rosetown (12 mg $\mathrm{kg}^{-1}$ ) had significantly higher concentration of $\mathrm{Cu}$ compared to other genotypes. Among the other subspecies (L. culinaris subsp. culinaris, L.culinaris subsp. orientalis, and $L$. culinaris subsp. tomentosus) genotypes IG72614and IG72616, belonging to the tomentosus subspecies, had equal and significantly higher concentrations of $\mathrm{Cu}\left(12 \mathrm{mg} \mathrm{kg}^{-1}\right)$ than other genotypes. Among the non-culinaris wild type genotypes IG110812 and IG72815 both had seed $\mathrm{Cu}$ concentrations of $6 \mathrm{mg} \mathrm{kg}^{-1}$, which was significantly greater than the other wild, genotypes. A serving of the lentil genotypes tested can provide $22-133 \%$ of the $\mathrm{Cu}$ RDA $(0.9$ mg) (Otten et al., 2006).

\section{Calcium concentration in cultivated and wild lentils}

Among all evaluated lentil genotypes, the mean $\mathrm{Ca}$ concentration was $339 \mathrm{mg} \mathrm{kg}^{-1}$ (Table 3). Mean Ca concentration among the 20 L. culinaris genotypes was 323 $\mathrm{mg} \mathrm{kg}^{-1}$, with the lowest concentration in Eston $\left(97 \mathrm{mg} \mathrm{kg}^{-1}\right)$ and the highest in Pennell $\left(536 \mathrm{mg} \mathrm{kg}^{-1}\right)$. Within L. culinaris subsp. culinaris genotypes Pennell (536 mg kg-1) had significantly higher concentration of $\mathrm{Ca}$ compared to other genotypes. Among the other subspecies (tomentosus, orientalis, odemensis) IG71594 (orientalis subspecies) had significantly higher concentration of $\mathrm{Ca}\left(534 \mathrm{mg} \mathrm{kg}^{-1}\right)$ than other genotypes. Among the non-culinaris wild type genotypes belonging to L. nigricans, IG72548 and IG72549, had significantly higher concentration of $\mathrm{Ca}\left(508 \mathrm{mg} \mathrm{kg}^{-1}\right.$ and $590 \mathrm{mg} \mathrm{kg}^{-1}$, respectively). Percent RDA (1000 mg) of Ca (Otten et al., 2006) ranged from 1-6\% per serving.

\section{Magnesium concentration in cultivated and wild lentils}

The mean $\mathrm{Mg}$ concentration among all tested lentil genotypes was $638 \mathrm{mg} \mathrm{kg}^{-1}$ (Table 3). Magnesium concentration ranged from 272 (CDC Redberry) to $892 \mathrm{mg} \mathrm{kg}^{-1}$ (Pennell) among the $20 \mathrm{~L}$. culinaris genotypes, with a mean of $616 \mathrm{mg} \mathrm{kg}^{-1}$. Within L. culinaris subsp. culinaris genotypes, the concentration of $\mathrm{Mg}$ in Penell seeds $\left(892 \mathrm{mg} \mathrm{kg}^{-1}\right.$ ) was significantly greater than other genotypes. Among the other subspecies (tomentosus, orientalis, odemensis) genotypes belonging to tomentosus subspecies IG72614 and IG72616 had significantly higher concentration of $\mathrm{Mg}\left(807 \mathrm{mg} \mathrm{kg}^{-1}\right.$ and $865 \mathrm{mg} \mathrm{kg}^{-1}$, respectively) than other genotypes. Among the non-culinaris wild types, L. lamottei, IG110813 had significantly higher concentration of $\mathrm{Mg}\left(839 \mathrm{mg} \mathrm{kg}^{-1}\right)$ compared to the other species. Percent RDA $(310 \mathrm{mg})$ of $\mathrm{Mg}$ (Otten et al., 2006) ranged from 9-29\% per serving.

\section{Discussion}

Lentil is a cool season food legume with a narrow genetic base, therefore genetic variability for individual traits is generally low (Eujayl et al., 1998). This low genetic variability is further exacerbated in breeding programs by the extensive use of superior genotypes with common ancestors (Kumar et al., 2004). Interspecific hybridization, either directly between cross compatible species or indirectly between cross-incompatible species using a bridge species, can be used in the genetic improvement of lentils (Kumar et al., 2011). This technique is utilized when a desirable 
Table 1. Brief description of plant materials used.

\begin{tabular}{|c|c|c|c|}
\hline Species & Genotype/accession & Remark & Origin \\
\hline L. culinaris subsp. culinaris & CDC Redberry & Small red cultivated type & University of Saskatchewan, Canada \\
\hline L. culinaris subsp. culinaris & CDC Rouleau & small red cultivated type & University of Saskatchewan, Canada \\
\hline L. culinaris subsp. culinaris & CDC Red Rider & medium red cultivated type & University of Saskatchewan, Canada \\
\hline L. culinaris subsp. culinaris & CDC Greenland & large green cultivated type & University of Saskatchewan, Canada \\
\hline L. culinaris subsp. culinaris & Barimasur-2 & small red cultivated type & BARI, Bangladesh \\
\hline L. culinaris subsp. culinaris & Barimasur-3 & small red cultivated type & BARI, Bangladesh \\
\hline L. culinaris subsp.culinaris & Eston & small green cultivated type & University of Saskatchewan, Canada \\
\hline L. culinaris subsp. culinaris & Pennell & large green cultivated type & WSU, USA \\
\hline L. culinaris subsp. orientalis & IG72594 & small seeded wild type & WSU, USA \\
\hline L. culinaris subsp. orientalis & IG72603 & small seeded wild type & WSU, USA \\
\hline L. culinaris subsp. orientalis & IG72618 & small seeded wild type & WSU, USA \\
\hline L. culinaris subsp. orientalis & IG72896 & small seeded wild type & WSU, USA \\
\hline L. ervoides & IG72815 & small seeded wild type & WSU, USA \\
\hline L. lamottei & IG110810 & small seeded wild type & WSU, USA \\
\hline L. lamottei & IG110812 & small seeded wild type & WSU, USA \\
\hline L. lamottei & IG110813 & small seeded wild type & WSU, USA \\
\hline L. nigricans & IG72548 & small seeded wild type & WSU, USA \\
\hline L. nigricans & IG72549 & small seeded wild type & WSU, USA \\
\hline
\end{tabular}

Table 2.Mean iron (Fe) and zinc ( $\mathrm{Zn})$ concentration and Percent Recommended Daily Allowance (\%RDA) of 26 lentil genotypes.

\begin{tabular}{|c|c|c|c|c|c|}
\hline $\begin{array}{l}\text { Genotype } \\
\text { (species) }\end{array}$ & Species & $\begin{array}{l}\text { Fe Concentration } \\
\left(\mathrm{mg} \cdot \mathrm{kg}^{-1}\right)\end{array}$ & $\% \mathrm{RDA}$ & $\begin{array}{l}\text { Zn concentration } \\
\left(\mathrm{mg} \cdot \mathrm{kg}^{-1}\right)\end{array}$ & $\%$ RDA \\
\hline CDC Redberry & L. culinaris subsp. culinaris & $91 \mathrm{a}$ & 51 & $37 \mathrm{c}, \mathrm{d}$ & 46 \\
\hline CDC Rosetown & L. culinaris subsp. culinaris & $82 \mathrm{a}, \mathrm{b}, \mathrm{c}$ & 46 & $51 \mathrm{a}$ & 64 \\
\hline CDC Rouleau & L. culinaris subsp. culinaris & $71 \mathrm{a}, \mathrm{b}, \mathrm{c}, \mathrm{d}, \mathrm{e}$ & 39 & $46 a, b$ & 58 \\
\hline CDC LeMay & L. culinaris subsp. culinaris & $68 \mathrm{~b}, \mathrm{c}, \mathrm{d}, \mathrm{e}, \mathrm{f}$ & 38 & $31 \mathrm{~d}, \mathrm{e}, \mathrm{f}, \mathrm{g}, \mathrm{h}$ & 39 \\
\hline CDC Red Rider & L. culinaris subsp. culinaris & $92 \mathrm{a}$ & 51 & $45 \mathrm{a}, \mathrm{b}$ & 56 \\
\hline CDC Greenland & L. culinaris subsp. culinaris & 64 c,d,e, f,g, & 36 & $43 \mathrm{~b}, \mathrm{c}$ & 54 \\
\hline Barimasur-2 & L. culinaris subsp. culinaris & 52 e,f,g,h,i,j & 29 & 33 d,e,f,g & 41 \\
\hline Barimasur-3 & L. culinaris subsp. culinaris & $46 \mathrm{f}, \mathrm{g}, \mathrm{i}, \mathrm{j}, \mathrm{k}, \mathrm{h}$ & 26 & $31 \mathrm{~d}, \mathrm{e}, \mathrm{f}, \mathrm{g}, \mathrm{h}$ & 39 \\
\hline Barimasur-4 & L. culinaris subsp. culinaris & $36 \mathrm{i}, \mathrm{j}, \mathrm{k}$ & 20 & $25 \mathrm{~h}, \mathrm{i}, \mathrm{j}$ & 31 \\
\hline Riveland & L. culinaris subsp. culinaris & $62 \mathrm{c}, \mathrm{d}, \mathrm{e}, \mathrm{f}, \mathrm{g}, \mathrm{h}$ & 34 & 30 e,f,g,h,i & 38 \\
\hline Eston & L. culinaris subsp. culinaris & 39 h,i,j,k & 22 & $17 \mathrm{k}$ & 21 \\
\hline Pennell & L. culinaris subsp. culinaris & $87 \mathrm{a}, \mathrm{b}$ & 48 & $36 \mathrm{c}, \mathrm{d}, \mathrm{e}$ & 45 \\
\hline IG72594 & L. culinaris subsp. orientalis & 54 e,f,g,h,i & 30 & 33 d,e,f & 41 \\
\hline IG72603 & L. culinaris subsp. orientalis & $34 \mathrm{j}, \mathrm{k}$ & 19 & $18 \mathrm{k}$ & 23 \\
\hline IG72830 & L. culinaris subsp. tomentosus & $26 \mathrm{k}$ & 14 & $17 \mathrm{k}$ & 21 \\
\hline IG72688 & L. culinaris subsp. odemensiss & $36 \mathrm{i}, \mathrm{j}, \mathrm{k}$ & 20 & $22 \mathrm{j}, \mathrm{k}$ & 28 \\
\hline IG72614 & L. culinaris subsp. tomentosus & $58 \mathrm{~d}, \mathrm{e}, \mathrm{f}, \mathrm{g}, \mathrm{h}$ & 32 & $43 \mathrm{~b}, \mathrm{c}$ & 54 \\
\hline IG72616 & L. culinaris subsp. tomentosus & $61 \mathrm{c}, \mathrm{d}, \mathrm{e}, \mathrm{f}, \mathrm{g}, \mathrm{h}$ & 34 & $36 \mathrm{c}, \mathrm{d}, \mathrm{e}$ & 45 \\
\hline IG72618 & L. culinaris subsp. orientalis & $43 \mathrm{~g}, \mathrm{~h}, \mathrm{i}, \mathrm{j}, \mathrm{k}$ & 24 & 24 i.j.k & 30 \\
\hline IG72896 & L. culinaris subsp. orientalis & $67 \mathrm{~b}, \mathrm{c}, \mathrm{d}, \mathrm{e}, \mathrm{f}$ & 37 & $26 \mathrm{~g}, \mathrm{~h}, \mathrm{i}, \mathrm{j}$ & 33 \\
\hline IG110810 & L. lamottei & 80 a,b,c,d & 44 & $26 \mathrm{f}, \mathrm{g}, \mathrm{h}, \mathrm{i}, \mathrm{j}$ & 33 \\
\hline IG110812 & L. lamottei & 64 b,c,d,e,f,g & 36 & $40 \mathrm{~b}, \mathrm{c}$ & 50 \\
\hline IG110813 & L. lamottei & 70 a,b,c,d,e & 39 & $31 \mathrm{~d}, \mathrm{e}, \mathrm{f}, \mathrm{g}, \mathrm{h}$ & 39 \\
\hline IG72548 & L. nigricans & $60 \mathrm{c}, \mathrm{d}, \mathrm{e}, \mathrm{f}, \mathrm{g}$ & 33 & $28 \mathrm{f}, \mathrm{g}, \mathrm{h}, \mathrm{i}, \mathrm{j}$ & 35 \\
\hline IG72549 & L. nigricans & $71 \mathrm{a}, \mathrm{b}, \mathrm{c}, \mathrm{d}, \mathrm{e}$ & 39 & $33 \mathrm{~d}, \mathrm{e}, \mathrm{f}$ & 41 \\
\hline IG72815 & L. ervoides & 65 b,c,d,e,f,g & 36 & $37 \mathrm{c}, \mathrm{d}, \mathrm{e}$ & 46 \\
\hline Mean & & 61 & 39 & 33 & 41 \\
\hline SE & & 2.4 & & 1.1 & \\
\hline SD & & 21.0 & & 10.1 & \\
\hline Range & & $26-92$ & $14-51$ & $17-51$ & $21-64$ \\
\hline
\end{tabular}

Means within a column followed by different letters are significantly different at $\mathrm{p}<0.05(\mathrm{n}=78)$, Percent RDA values were calculated with daily requirement of 18 mg of Fe and 8 $\mathrm{mg}$ of $\mathrm{Zn}$ (females, age 19+ years) (Otten et al., 2006). Percent RDAs were calculated based on the serving size of $100 \mathrm{~g}$ of cooked lentil. 
Table 3. Mean copper $(\mathrm{Cu})$, calcium $(\mathrm{Ca})$ and magnesium $(\mathrm{Mg})$ concentration and Percent Recommended Dietary Allowance (\%RDA) of 26 lentil genotypes.

\begin{tabular}{|c|c|c|c|c|c|c|c|}
\hline Genotype & Species & $\begin{array}{l}\mathrm{Cu} \\
\left(\mathrm{mg} \cdot \mathrm{kg}^{-1}\right)\end{array}$ & $\%$ RDA & $\begin{array}{l}\mathrm{Ca} \\
\left(\mathrm{mg} \cdot \mathrm{kg}^{-1}\right)\end{array}$ & $\% \mathrm{AI}$ & $\begin{array}{l}\mathrm{Mg} \\
\left(\mathrm{mg} \cdot \mathrm{kg}^{-1}\right)\end{array}$ & $\%$ RDA \\
\hline CDC Redberry & $\begin{array}{l}\text { L. culinaris subsp. } \\
\text { culinaris }\end{array}$ & $10 \mathrm{~b}$ & 111 & $323 \mathrm{~b}, \mathrm{c}, \mathrm{d}, \mathrm{e}$ & 3 & 2721 & 9 \\
\hline CDC Rosetown & $\begin{array}{l}\text { L. culinaris subsp. } \\
\text { culinaris }\end{array}$ & $12.0 \mathrm{a}$ & 133 & $257 \mathrm{e}, \mathrm{f}$ & 3 & $423 \mathrm{j}, \mathrm{k}, 1$ & 14 \\
\hline CDC Rouleau & $\begin{array}{l}\text { L. culinaris subsp. } \\
\text { culinaris }\end{array}$ & $9.0 \mathrm{~b}, \mathrm{c}, \mathrm{d}$ & 100 & 318 b,c,d,e & 3 & $556 \mathrm{~h}, \mathrm{i}, \mathrm{j}$ & 18 \\
\hline CDC LeMay & $\begin{array}{l}\text { L. culinaris subsp. } \\
\text { culinaris }\end{array}$ & $7 \mathrm{~d}, \mathrm{e}, \mathrm{f}$ & 78 & $409 \mathrm{~b}$ & 4 & 842 a,b,c & 27 \\
\hline CDC Red Rider & $\begin{array}{l}\text { L. culinaris subsp. } \\
\text { culinaris }\end{array}$ & $10 \mathrm{~b}$ & 111 & $361 \mathrm{~b}, \mathrm{c}$ & 4 & 656 d,e,f,g,h,i & 21 \\
\hline CDC Greenland & $\begin{array}{l}\text { L. culinaris subsp. } \\
\text { culinaris }\end{array}$ & $9.0 \mathrm{~b}, \mathrm{c}, \mathrm{d}$ & 100 & $205 \mathrm{f}, \mathrm{g}$ & 2 & $610 \mathrm{f}, \mathrm{g}, \mathrm{h}, \mathrm{i}$ & 20 \\
\hline Barimasur-2 & $\begin{array}{l}\text { L. culinaris subsp. } \\
\text { culinaris }\end{array}$ & $4.0 \mathrm{~g}, \mathrm{~h}, \mathrm{i}, \mathrm{j}$ & 44 & 337 b,c,d,e & 3 & 697 c,d,e,f,g,h,i & 22 \\
\hline Barimasur-3 & $\begin{array}{l}\text { L. culinaris subsp. } \\
\text { culinaris }\end{array}$ & 4.0i,j,k & 44 & 314 b,c,d,e & 3 & 707 b,c,d,e,f,g,h & 23 \\
\hline Barimasur-4 & $\begin{array}{l}\text { L. culinaris subsp. } \\
\text { culinaris }\end{array}$ & $6.0 \mathrm{f}, \mathrm{g}, \mathrm{h}, \mathrm{i}$ & 67 & 344 b,c,d,e & 3 & 662 d,e,f,g,h,i & 21 \\
\hline Riveland & $\begin{array}{l}\text { L. culinaris subsp. } \\
\text { culinaris }\end{array}$ & $8.0 \mathrm{~b}, \mathrm{c}, \mathrm{d}$ & 89 & 355 b,c,d & 4 & $5371 \mathrm{i}, \mathrm{j}$ & 17 \\
\hline Eston & $\begin{array}{l}\text { L. culinaris subsp. } \\
\text { culinaris }\end{array}$ & $4.0 \mathrm{~g}, \mathrm{~h}, \mathrm{i}, \mathrm{j}$ & 44 & $97 \mathrm{~h}$ & 1 & $3311 \mathrm{k}, 1$ & 11 \\
\hline Pennell & $\begin{array}{l}\text { L. culinaris subsp. } \\
\text { culinaris }\end{array}$ & $7.0 \mathrm{~d}, \mathrm{e}, \mathrm{f}$ & 78 & $536 \mathrm{a}$ & 5 & 892 a & 29 \\
\hline IG72594 & $\begin{array}{l}\text { L. culinaris subsp. } \\
\text { orientalis }\end{array}$ & $3.0 \mathrm{j}, \mathrm{k}$ & 33 & $534 \mathrm{a}$ & 5 & $584 \mathrm{~g}, \mathrm{~h}, \mathrm{i}, \mathrm{j}$ & 19 \\
\hline IG72603 & $\begin{array}{l}\text { L. culinaris subsp. } \\
\text { orientalis }\end{array}$ & $3.0 \mathrm{j}, \mathrm{k}$ & 33 & 313 b,c,d,e & 3 & $581 \mathrm{~g}, \mathrm{~h}, \mathrm{i}, \mathrm{j}$ & 19 \\
\hline IG72830 & $\begin{array}{l}\text { L. culinaris subsp. } \\
\text { tomentosus }\end{array}$ & $4.0 \mathrm{j}, \mathrm{k}$ & 44 & $112 \mathrm{~g}, \mathrm{~h}$ & 1 & $3751 \mathrm{k}, 1$ & 12 \\
\hline IG72688 & $\begin{array}{l}\text { L. culinaris subsp. } \\
\text { odemensiss }\end{array}$ & $3.0 \mathrm{j}, \mathrm{k}$ & 33 & 352 b,c,d,e & 4 & 643 e,f,g,h,i & 21 \\
\hline IG72614 & $\begin{array}{l}\text { L. culinaris subsp. } \\
\text { tomentosus }\end{array}$ & $6.0 \mathrm{e}, \mathrm{f}$ & 67 & $304 \mathrm{c}, \mathrm{d}, \mathrm{e}$ & 3 & 807 a,b,c,d & 26 \\
\hline IG72616 & $\begin{array}{l}\text { L. culinaris subsp. } \\
\text { tomentosus }\end{array}$ & $6.0 \mathrm{e}, \mathrm{f}, \mathrm{g}$ & 67 & 341 b,c,d,e & 3 & $865 \mathrm{a}, \mathrm{b}$ & 28 \\
\hline IG72618 & $\begin{array}{l}\text { L. culinaris subsp. } \\
\text { orientalis }\end{array}$ & $4.0 \mathrm{~h}, \mathrm{i}, \mathrm{j}$ & 44 & 264 d,e,f & 3 & $540 \mathrm{i}, \mathrm{j}$ & 17 \\
\hline IG72896 & $\begin{array}{l}\text { L. culinaris subsp. } \\
\text { orientalis }\end{array}$ & $3.0 \mathrm{j}, \mathrm{k}$ & 33 & 368 b,c & 4 & 732 a,b,c,d,e,f,g & 24 \\
\hline IG110810 & L. lamottei & $2.0 \mathrm{k}$ & 22 & $357 \mathrm{~b}, \mathrm{c}, \mathrm{d}$ & 4 & 754 a,b,c,d,e,f & 24 \\
\hline IG110812 & L. lamottei & $6.0 \mathrm{e}, \mathrm{f}, \mathrm{g}, \mathrm{h}$ & 67 & $311 \mathrm{c}, \mathrm{d}, \mathrm{e}$ & 3 & 789 a,b,c,d,e & 25 \\
\hline IG110813 & L. lamottei & $5.0 \mathrm{e}, \mathrm{f}$ & 56 & $434 \mathrm{c}, \mathrm{d}, \mathrm{e}$ & 4 & 839 a,b,c,d & 27 \\
\hline IG72548 & L. nigricans & $4.0 \mathrm{i}, \mathrm{j}, \mathrm{k}$ & 44 & $508 \mathrm{a}$ & 5 & 738 a,b,c,d,e,f,g & 24 \\
\hline IG72549 & L. nigricans & $3.0 \mathrm{j}, \mathrm{k}$ & 33 & $590 \mathrm{a}$ & 6 & $445 \mathrm{j}, \mathrm{k}$ & 14 \\
\hline IG72815 & L. ervoides & $6.0 \mathrm{e}, \mathrm{f}$ & 67 & $292 \mathrm{c}, \mathrm{d}, \mathrm{e}, \mathrm{f}$ & 3 & $756 \mathrm{a}, \mathrm{b}, \mathrm{c}, \mathrm{d}, \mathrm{e}, \mathrm{f}$ & 24 \\
\hline Mean & & 6 & 67 & 339 & 3 & 638 & 21 \\
\hline SE & & 0.3 & & 13.9 & & 21 & \\
\hline SD & & 2.7 & & 122 & & 185.45 & \\
\hline Range & & $2-12$ & 22-133 & $97-590$ & $1-6$ & $272-892$ & $9-29$ \\
\hline
\end{tabular}

Means within a column followed by different letters are significantly different at $\mathrm{p}<0.05(\mathrm{n}=78)$, Percent RDA were calculated with daily requirement of $900 \mu \mathrm{g}$ for $\mathrm{Cu}$, $1000 \mathrm{mg}$ for $\mathrm{Ca}$, and $310 \mathrm{mg}$ for $\mathrm{Mg}$ (females age 19+) (Otten et al., 2006). Percent RDAs were calculated based on the serving size of $100 \mathrm{~g}$ of cooked lentil. For Ca, Adequate Intake (AI) values are available, not the RDA (Otten et al., 2006).

characteristic is present in another related or crossable species (Tullu et al., 2011). Biofortification for mineral traits is a priority research area in food legumes (including lentil) (Grusak, 2009; Thavarajah et al., 2009, 2011; Johnson et al., 2013; Iqbal et al., 2006; Hunt 2003). Identifying and utilizing donors with alleles that result in high seed concentrations of mineral nutrients is required for the successful development of biofortified cultivars. This study has documented that the Lens wild species and subspecies that were evaluated are usually an inferior source for seed mineral concentration compared to cultivated types. However, generation of transgressive segregants, due to the accumulation of additive genes, from crosses between or within different species or subspecies is not ruled out..The present study may provide suitable genotypes to make crosses to develop breeding populations. The selection of genotypes based on concentration of micronutrients ( $\mathrm{Fe}, \mathrm{Zn}, \mathrm{Cu}, \mathrm{Ca}, \mathrm{Mg}$ ) (Table 2 and Table 3) could be utilized to develop intraspecific or interspecific recombinant inbred line populations to identify and map QTL associated with seed mineral concentrations. 
While making interspecific crosses the cross-compatibility has to be taken into consideration. The primary gene pool members are easily cross-compatible (Lens culinaris subsp. culinaris, Lens culinaris subsp. odemensis, Lens culinaris subsp. orientalis, Lens culinaris subsp. tomentosus) (Ferguson et al., 2000). Crossing between primary and secondary/tertiary gene pools members ( $L$. ervoides, $L$. lamottei, L. nigricans) may require the use of tissue culture based techniques, such as embryo rescue, or the use of bridge species (Ferguson et al., 2000).

In the present study, significant variation in mineral $(\mathrm{Fe}$, $\mathrm{Zn}, \mathrm{Cu}, \mathrm{Ca}, \mathrm{Mg}$ ) concentration was observed. Similarly, Karaköy et al., (2012) studied the mineral status of Turkish lentil landraces and cultivars in lentil. They reported $\mathrm{Fe}$ concentration from 49.40 to $81.39 \mathrm{mg} \mathrm{kg}^{-1}$. The concentrations reported for $\mathrm{Zn}, \mathrm{Cu}, \mathrm{Ca}$, and $\mathrm{Mg}$ were 46.90 $73.10 \mathrm{mg} \mathrm{kg}^{-1}, 9.10-16.92 \mathrm{mg} \mathrm{kg}^{-1}, 480-1280 \mathrm{mg} \mathrm{kg}^{-1}$ and $850-1260 \mathrm{mg} \mathrm{kg}^{-1}$, respectively. Similarly, Solanki et al., (1999) evaluated improved lentil cultivars in India. They reported $\mathrm{Fe}$ concentration from 80 to $92 \mathrm{mg} \mathrm{kg}^{-1}$ and $\mathrm{Ca}$ concentration from 1150 to $1650 \mathrm{mg} \mathrm{kg}^{-1}$. The Ca concentrations Solanki et al., (1999) reported were higher than those from the present study, possibly due to thegenotypic differences in Indian lentil cultivars and or different field soilconditions. Thavarajah et al., (2009) reported $\mathrm{Fe}$ and $\mathrm{Zn}$ concentrations of 73-90 and 44-54 $\mathrm{mg} \mathrm{kg}^{-}$ 1 , respectively, in a set of lentil cultivars by analyzing seeds harvested from regional varietal trials of 19 genotypes from 9 locations in Saskatchewan, Canada over 2 years. The present study demonstrated more variation for these two micronutrients, which is attributed to the inclusion of related species in addition to L. culinaris. Zia-Ul-Haq et al., (2011) evaluated four improved lentil cultivars from Pakistan for different micronutrients and reported that $\mathrm{Fe}, \mathrm{Zn}, \mathrm{Cu}$, and $\mathrm{Ca}$ concentrationranged from 27-32, 39-44, 89-99, and 1180$1210 \mathrm{mg} \mathrm{kg}^{-1}$, respectively. In a study comparing micronutrient concentrations in different legumes, Iqbal et al., (2006) found that $\mathrm{Fe}, \mathrm{Zn}, \mathrm{Cu}, \mathrm{Ca}$ and $\mathrm{Mg}$ concentration was $31,44,99,1200$, and $45 \mathrm{mg} \mathrm{kg}^{-1}$, respectively, in lentil. In these studies, the reported $\mathrm{Fe}$ concentration was very low and the $\mathrm{Ca}$ and $\mathrm{Cu}$ concentrations were very high compared to the data presented here. The differences may be due to the fact that seeds were not from the single uniform trials, as they did not report the methods of growing the plants. In addition, differences might be due to their use of the less sensitive or accurate flame/graphite atomic absorption spectrophotometer (AAS), compared to the more sensitive ICP-OES, to determine micronutrients. AAS is more vulnerable to physical and chemical interferences than is ICP-OES. Alghamdi et al., (2014) studied 35 advanced breeding lines of cultivated lentilin Saudi Arabia from a field trial over two years and reported concentrations for $\mathrm{Mg}$ (1261-1573 $\mathrm{mg} \mathrm{kg}$ $\left.{ }^{1}\right), \mathrm{Ca}\left(64.9-84 \mathrm{mg} \mathrm{kg}^{-1}\right), \mathrm{Fe}\left(65.7-85.7 \mathrm{mg} \mathrm{kg}^{-1}\right)$, Zn (26.3 $\left.45.1 \mathrm{mg} \mathrm{kg}^{-1}\right)$, and $\mathrm{Cu}\left(8.6-13.7 \mathrm{mg} \mathrm{kg}^{-1}\right)$. This corresponds closely to the concentrations of $\mathrm{Fe}, \mathrm{Zn}$, and $\mathrm{Cu}$ but not for $\mathrm{Mg}$ and $\mathrm{Ca}$ concentration reported in the present study.

The Food and Nutrition Board of the Institute of Medicine, The National Academies, USA established percent recommended dietary allowance (RDA) for the minerals (Otten et al., 2006). The RDA is the average recommended daily level of intake of a particular nutrient that is sufficient to meet the nutrient requirements of nearly all (97-98\%) healthy people (Otten et al., 2006). The values vary by age and gender and in this study, the RDA used was for females, 19 to 50 years old.This category was chosen as the daily intake requirements for most minerals were higher for adult females than adult males and children. Percent RDA values were calculated based on a $100 \mathrm{~g}$ serving size of cooked lentils for each of the minerals (Otten et al., 2006). A considerable proportion (for Fe 14-51\%, for $\mathrm{Zn} 21-64 \%$, for $\mathrm{Cu} 22-133 \%$, Mg 9-29\%) of RDA for minerals would be obtained from consuming $100 \mathrm{~g}$ of cooked lentils (Table 2 and 3). This is similar to data reported in previous studies (Thavarajah et al., 2009, 2011). Percent RDA of Ca was only $1-6 \%$ for the genotypes we evaluated, indicating that lentil as a not good source $\mathrm{Ca}$. Developing lentil varieties with high concentrations of $\mathrm{Fe}$ and $\mathrm{Zn}$ would be especially beneficial for Asian and African countries where 40-45\% of school-age children are Fe- and Zn-deficient (de Benoist et al., 2008).

\section{Materials and Methods}

\section{Chemicals}

Chemical reagents and standards used for mineral digestion and analytical determinations were purchased from Alfa Aesar, VWR International and Sigma-Aldrich Co. (St. Louis, MO, USA) and used without further purification. Water (distilled and deionized; $\mathrm{ddH}_{2} \mathrm{O}$ ) was purified by a Milli-Q Water System (Millipore, Milford, MA, USA) to a resistance of $18.2 \mathrm{M} \Omega \mathrm{cm}$ or greater.

\section{Plant materials}

The experimental genotypes included 12 L. culinaris subsp. culinaris, 4 L. culinaris subsp. orientalis, 3 L. culinaris subsp. tomentosus, $1 \mathrm{~L}$. culinaris subsp. odemensis, $1 \mathrm{~L}$. ervoides, 3 L. lamottei and 2 L. nigricans genotypes (Table 1). This set of genotypes was selected as it represents different market classes of cultivated lentil as well as the subspecies of L. culinaris and the wild relatives. The seeds were obtained from the USDA-ARS Grain Legume Genetics and Physiology Research Unit, WSU, Pullman, Washington, USA and maintained as single plant selections in the former Pulse Quality Laboratory, NDSU, Fargo, ND, USA.

\section{Greenhouse experiment}

Ten surface sterilized seeds from each lentil genotype were placed in sterile petri dishes with absorbent filter paper saturated with Millipore filtered water. The petri dishes were placed in the dark at room temperature $\left(22^{\circ} \mathrm{C}\right)$. Every second day, the absorbent paper was saturated with $2-3 \mathrm{~mL}$ of Millipore water. Plastic pots $(15.25 \mathrm{~cm})$ were filled with approximately $300 \mathrm{~g}$ of a peat-perlite-vermiculite mixture (Sunshine Grow Mix Number 1, Sun Gro Horticulture Canada Inc., ON, Canada) and saturated with deionized water. The pots were allowed to drain overnight, and then the weight of each pot recorded. At seeding, three germinated seeds of each lentil genotype were sown in pots at $70 \%$ field capacity. A total of 78 pots were seeded: three replicates of the 26 genotypes with randomization among the pots following a completely randomized design. Greenhouse conditions were as follows: day/night temperatures of $22{ }^{\circ} \mathrm{C} /$ $16{ }^{\circ} \mathrm{C}$; photosynthetically active radiation levels of $300 \mu \mathrm{mol}$ $\mathrm{m}^{-2} \cdot \mathrm{s}^{-1}$ using a $16 \mathrm{~h}$ photoperiod beginning at 0600 local time, and $50-60 \%$ relative humidity. Pots were watered to approximately $70 \%$ of free draining moisture concentration every day. Every two weeks, $250 \mathrm{~mL}$ of nutrient solution was added to each pot. Nutrient concentrations of the allpurpose Plants-Prod 20-20-20 Classic fertilizer solution (Plant Products Co. Ltd., Brampton, ON, Canada) were 20\% total N, 20\% total P, $20 \%$ soluble K, $0.02 \%$ B, $0.05 \%$ chelated $\mathrm{Cu}, 0.1 \%$ chelated $\mathrm{Fe}, 0.05 \% \mathrm{Mo}, 0.05 \% \mathrm{Zn}$, and 
1\% EDTA. Plants were thinned to two per pot after one week. Plants were harvested at physiological maturity and threshed individually. Seeds were ground using a stainless steel coffee grinder to obtain fine quality flour.

\section{Mineral concentration}

Mineral (Fe, $\mathrm{Zn}, \mathrm{Cu}, \mathrm{Ca}, \mathrm{Mg}$ ) concentrations in lentil seeds were determined using a previously described modified $\mathrm{HNO}_{3}-\mathrm{H}_{2} \mathrm{O}_{2}$ method (Alcok et al., 1987; Thavarajah et al., 2009). Finely ground seed samples $(500 \mathrm{mg})$ were placed in individual digestion tubes. Six $\mathrm{mL}$ of concentrated $(70 \%)$ nitric acid $\left(\mathrm{HNO}_{3}\right)$ was added to each digestion tube. The digestion tubes were placed in a $90{ }^{\circ} \mathrm{C}$ digestion block for one hour, and they were shaken at 15 and 45 minutes. Three $\mathrm{mL}$ of $30 \%$ w/w hydrogen peroxide $\left(\mathrm{H}_{2} \mathrm{O}_{2}\right)$ was then added to each tube. The tubes were kept for $15 \mathrm{~m}$ at $90{ }^{\circ} \mathrm{C}$. Finally, 3 $\mathrm{mL}$ of $6 \mathrm{M}$ hydrochloric acid $(\mathrm{HCl})$ was added to each digestion tube, and the tubes were kept in the digestion block for 5 minutes. Upon complete digestion (the time required for complete digestion was determined in earlier laboratory experiments, the complete digestion is indicated by the discontinuation of brown smoke coming from the digestion tubes), the tubes were removed from the digestion block, the volume was adjusted to $10 \mathrm{~mL}$, and then filtered (Whatman No. 1 filter papers) using a vacuum system (Gardener Denver Thomas Inc., Welch Vacuum Technologies, LA, USA). Mineral concentrations of the filtrates were measured using inductively coupled plasma-optical emission spectroscopy (ICP-OES); ICP-6500 Duo, Thermo Fisher Scientific, Pittsburg, PA, USA). Measurements of total minerals were validated using National Institute of Standards and Technology (NIST) standard reference material (SRM) 1576a (wheat flour; $[\mathrm{Fe}]=14.11 \pm 0.13 \mathrm{mg} \mathrm{kg}^{-1},[\mathrm{Zn}]=11.61 \pm 0.26 \mathrm{mg}$ $\mathrm{kg}^{-1},[\mathrm{Ca}]=191.4 \pm 3.3 \mathrm{mg} \mathrm{kg}{ }^{-1},[\mathrm{Mg}]=398 \pm 12 \mathrm{mg} \mathrm{kg}^{-1}$, $[\mathrm{Cu}]=2.03 \pm 0.14 \mathrm{mg} \mathrm{kg}^{-1}$ ). Calibration curves for $\mathrm{Fe}, \mathrm{Zn}$, and $\mathrm{Cu}$ concentration were made using serial dilutions from 0.5 to $50.0 \mathrm{mg} \mathrm{L}^{-1}$. The detection limit was $5 \mu \mathrm{g} \mathrm{L}^{-1}$. Calibration curves for $\mathrm{Ca}$ and $\mathrm{Mg}$ concentration were made using serial dilutions from 10 to $500 \mathrm{mg} \mathrm{L}^{-1}$.

\section{Statistical analysis}

The experimental design was a complete randomized design (CRD) with three replicates of 26 Lens genotypes $(n=78)$. Analysis of variance was performed using the General Linear Model (PROC GLM) of SAS version 9.3 (SAS Institute, 2009). Means were separated using Fisher's protected least significant difference (LSD) at $P<0.05$.

\section{Conclusion}

Lentils are an integral part of peoples' diets in many countries in Asia, including Bangladesh, Nepal, India, and Pakistan. People living in these areas are affected with mineral deficiencies, particularly iron deficiency anemia. Biofortification of minerals in lentil will have a positive impact on maternal and child health in these mineral deficiency affected areas. This study reports on the mineral status of different Lens species for $\mathrm{Fe}, \mathrm{Zn}, \mathrm{Cu}, \mathrm{Ca}$ and $\mathrm{Mg}$. This information could be for breeding programs to help direct choices of parents for intra- or interspecific hybridization. While this study is not exhaustive, it may serve as a caution for potential linkage drag on seed mineral nutrient concentration when introgressing a desired trait, e.g. disease resistance, from a Lens subspecies or wild relative into current cultivars. Utilization of different genotypes with very high and very low mineral concentrations may be used to develop mapping populations used to identify QTL associated with these micronutrients in lentil. Genomic approaches (Kaur et al., 2011; Verma et al., 2013; Sharpe et al., 2013; Wong et al., 2015) could be used to map or tag genes involved in seed mineral concentration in lentil and for precise introgression of novel traits from the Lens species and wild relatives.

\section{Acknowledgements}

Financial support for this research was provided by the Northern Pulse Growers Association, USA to Dr. Dil Thavarajah. We thank the Indian Council of Agricultural Research (ICAR), New Delhi, India for awarding an ICARInternational Fellowship to Debjyoti Sen Gupta.

\section{References}

Alcock NW (1987) A hydrogen-peroxide digestion system for tissue trace-metal analysis. Biol Trace Elem Res. 13:363-370.

Alghamdi SS, Khan AM, Ammar MH, El-Harty EH, Migdadi HM, El-Khalik SMA, Al-Shameri AM, Javed MM, Al-Faifi SA (2013) Phenological, nutritional and molecular diversity assessment among 35 introduced lentil (Lens culinaris Medik.) genotypes grown in Saudi Arabia. Int J Mol Sci. 15:277-295.

Bouis H, Low J, McEwan M, Tanumihardjo S (2013) Biofortification: Evidence and lessons learned linking agriculture and nutrition. FAO, WHO. [Online] Available at:

http://www.fao.org/fileadmin/user_upload/agn/pdf/Bioforti fication_paper.pdf. Accessed on 27 Oct 2015.

de Benoist B, McLean E, Egli I, Cogswell M (2008) Worldwide prevalence of anaemia 1993-2005. World Health Organization. [Online] Available at: http://apps.who.int/iris/bitstream/10665/43894/1/97892415 96657_eng.pdf. Accessed on 27 Oct 2015.

Eujayl I, Baum M, Powell W, Erskine W, Pehu, E (1998) A genetic linkage map of lentil (Lens sp.) based on RAPD and AFLP markers using recombinant inbred lines. Theor Appl Genet. 97:83-89.

Ferguson ME, Maxted N, van Slageren M, Robertson LD (2000) A re-assessment of the taxonomy of Lens Mill. (Leguminosae, Papilionoideae, Vicieae). Bot J Linn Soc 133:41-59.

Fiala JV, Tullu A, Banniza S, Séguin-Swartz G, Vandenberg A (2009) Interspecies transfer of resistance to anthracnose in lentil (Medic.). Crop Sci. 49:825-830.

Grusak M (2009) Nutritional and health-beneficial quality. In: Erskine W (ed) The Lentil: Botany, Production and Uses. Oxford: CAB International, pp. 368-390.

Hunt JR (2003) Bioavailability of iron, zinc, and other trace minerals from vegetarian diets. Am J Cln Nutr. 78:633S-639S.

IFAD/FAO/WFP (2011) The State of Food Insecurity in the World. [online] Available at: http://www.fao.org/3/ai4646e.pdf. Aaccessed on 27 Oct 2015.

Iqbal A, Khalil IA, Ateeq N, Khan MS (2006) Nutritional quality of important food legumes. Food Chem. 97:331-335.

Johnson C, Thavarajah D, Combs GF Jr, Thavarajah P (2013) Lentil (Lens culinaris L.): a prebiotic rich whole food legume. Food Res Int. 52:107-113. 
Karaköy T, Erdem H, Baloch FS, Toklu F, Eker S, Kilian B, Özkan H (2012) Diversity of macro-and micronutrients in the seeds of lentil landraces. The Sci World J. doi:10.1100/2012/710412 accessed on 27 Oct 2015.

Kaur S, Cogan NO, Pembleton LW, Shinozuka M, Savin KW, Materne M, Forster JW (2011) Transcriptome sequencing of lentil based on second-generation technology permits large-scale unigene assembly and SSR marker discovery. BMC Genomics 12:265.

Kumar S, Gupta S, Chandra S, Singh BB (2004) How wide is the genetic base of pulse crops? In: Ali, M, ed. Pulses in new perspective. Kanpur, India: Indian Society of Pulses Research and Development, 211-22. [Online] Available at http://eprints.icrisat.ac.in/9335/1/Howwide_211-

221_20004.pdf accessed on 27 Oct 2015.

Kumar S, Imtiaz M, Pratap A, Gupta S (2011) Distant hybridization and alien gene introgression in food legumes. In: Pratap A,Kumar J, eds. Biology and Breeding of Food Legumes. Oxford, UK: CAB International, 81-110.

Ladizinsky G, Cohen D, Muehlbauer FJ (1985) Hybridization in the genus Lens by means of embryo culture. Theor Appl Genet. 70:97-101.

Otten JJ, Hellwig JP, Meyers LD (2006) Dietary Reference Intakes: The Essential Guide to Nutrient Requirements. Washington, DC, USA: The National Academies Press.

Pfeiffer WH, McClafferty B (2007) HarvestPlus: Breeding crops for better nutrition. Crop Sci. 47:89-105.

Rohlf FJ (2009) NTSYS-pc numerical taxonomy and multivariate analysis system, version 2.21q Exeter Publ, New York, USA.

SAS Institute Inc (2008) SAS/SATS 9.3 User's Guide; SAS Institute: Cary, North Carolina.

Sharpe AG, Ramsay L, Sanderson LA, Fedoruk MJ, Clarke WE, Li R, Kagale S, Vijayan P, Vandenberg A, Bett KE (2013) Ancient orphan crop joins modern era: gene-based SNP discovery and mapping in lentil. BMC Genomics 14:192. accessed on 27 Oct 2015.

Solanki IS, Kapoor AC, Singh, U (1999) Nutritional parameters and yield evaluation of newly developed genotypes of lentil (Lens culinaris Medik.). Plant Food Human Nutr. 54:79-87.

Thavarajah D, Thavarajah P, Sarker A, Vandenberg A (2009) Lentils (Lens culinaris Medikus Subspecies culinaris): a whole food for increased iron and zinc intake. J Agr Food Chem. 57:5413-5419.
Thavarajah D, Thavarajah P, Wejesuriya A, Rutzke M, Glahn RP, Combs GF Jr, Vandenberg, A 2011. The potential of lentil (Lens culinaris L.) as a whole food for increased selenium, iron, and zinc intake: preliminary results from a 3 year study. Euphytica 180:123-128.

Tullu A, Diederichsen A, Suvorova G, Vandenberg A (2011) Genetic and genomic resources of lentil: status, use and prospects. Plant Genet Resour. 9:19-29.

U.S. Department of Agriculture. Agricultural Research Service (2015) USDA National Nutrient Database for Standard Reference, 27. Available at http://ndb.nal.usda.gov/ndb/foods/show/4782?manu=\&fgcd $=$ accessed on 27 Oct 2015 .

Welch RM, Graham RD (1999) A new paradigm for world agriculture: meeting human needs: productive, sustainable and nutritious. Field Crop Res. 60:1-10.

White PJ, Broadley MR (2009) Biofortification of crops with seven mineral elements often lacking in human diets: iron, zinc, copper, calcium, magnesium, selenium and iodine. New Phytol. 182:49-84. doi: 10.1111/j.14698137.2008.0273

Wong MM, Gujaria-Verma N, Ramsay L, Yuan HY, Caron C, Diapari M, Vandeberg A, Bett K E (2015) Classification and characterization of species within the genus Lens using genotyping-by-sequencing (GBS). PloS One. 10, e0122025 accessed on 27 Oct 2015.

Zia-Ul-Haq M, Ahmad S, Shad MA, Iqbal S, Qayum M, Ahmad A, Luthria DL, Amarowicz, R (2011) Compositional studies of lentil (Lens culinaris Medik.) cultivars commonly grown in Pakistan. Pakistan J Bot. 43:1563-1567. 